\title{
Interférométrie optique et observations astrophysiques
}

\author{
Pierre KERN et Jean-Baptiste LE BOUQUIN \\ UJF-Grenoble 1 / CNRS-INSU, Institut de Planétologie et d'Astrophysique de Grenoble (IPAG), UMR 5274 \\ Pierre.Kern@obs.vif-grenoble.fr \\ Jean-Baptiste.Lebouquin@obs.ujf-grenoble.fr
}

Depuis la fin des années 90, les astronomes utilisent l'interférométrie de manière régulière comme outil d'observation pour dépasser la limite de résolution des plus grands télescopes actuels. Après une période de validation au moyen de premiers prototypes de réseaux de télescopes, l'effort est porté maintenant sur l'optimisation de l'instrumentation utilisée au foyer des réseaux opérationnels. Des progrès technologiques sont attendus, en particulier en matière de détection, pour étendre le domaine d'application de cette technique très puissante.

\section{L'interférométrie :}

\section{un outil puissant au service de l'astrophysique}

L'observation astrophysique à haute résolution angulaire est théoriquement limitée par la diffraction de la pupille d'entrée de l'instrument. Cette limite est donnée par le rapport de la longueur d'onde d'observation au diamètre du télescope (a $\sim \lambda / D)$. Ainsi un télescope unique de la classe 10 mètres est capable d'atteindre une résolution de 50 mas (millisecondes d'arc) dans le proche infrarouge $(1 \mu \mathrm{m})$. De nombreuses questions de l'astrophysique nécessitent un meilleur pouvoir de résolution, par exemple pour réaliser l'image de la surface des étoiles les plus proches, celles-ci ayant un diamètre au plus de 2 mas, ou bien pour l'étude de zones de formation de planètes se déployant à des distances comprises entre 0,1 et 100 mas de l'étoile hôte.

Pour gagner le facteur 100 manquant, il faut augmenter la dimension de la pupille d'entrée de façon significative. Untel gain n'est possible que par le biais de pupilles synthétiques géantes réalisées au moyen de réseaux de télescopes. Un réseau de télescopes déployés sur des bases atteignant 100 mètres, permettra une résolution 10 fois supérieure aux mêmes longueurs d'onde.

Le signal interférométrique obtenu par recombinaison de la lumière provenant d'un même objet observé par un réseau de télescopes est une somme de systèmes de franges d'interférences, correspondant à chaque paire de télescopes. Les visibilités complexes (module et phase) de ces franges codent l'information correspondant à la structure de cet objet. La mesure du contraste des franges pour une paire de télescopes donnée fournit une mesure à la fréquence spatiale correspondante. Pour une séparation croissante entre les deux télescopes, une étoile double produit des systèmes de franges dont le contraste varie entre un maximum et un minimum en fonction de la séparation de ces télescopes. Ce signal sinusoïdal correspond à la transformée de Fourier de l'objet observé, l'étoile double observée ici correspondant à un double Dirac. Dans le cas d'un disque de brillance uniforme on obtient ainsi un profil de Bessel. La méthode décrite par le théorème de Van Cittert Zernicke, est maintenant éprouvée et permet de reconstruire l'image d'objets plus complexes. La complexité accessible est en définitive directement reliée au nombre de points de mesure. Ce nombre de termes de Fourier mesurables (fréquences spatiales) correspond au nombre de bases et évolue par conséquent approxi- mativement comme le nombre de télescopes du réseau. Le nombre de bases peut aussi être augmenté en tirant profit de la rotation de la Terre qui a pour effet de changer la projection des bases de l'interféromètre.

On retiendra que les éléments de dimensionnement importants d'un interféromètre sont les suivants :

- La séparation maximum entre les télescopes donne la résolution maximum du réseau dans la direction de cette base. À ce jour les plus grands interféromètres optiques et infrarouges ont des bases de plusieurs centaines de mètres, atteignant le kilomètre pour des installations prototypes.

- Un grand nombre de télescopes permet d'améliorer la capacité de reconstruction d'image, les plus grands réseaux comportant jusqu'à 8 télescopes sur leur site.

- Le diamètre des télescopes détermine la sensibilité du système. Les plus grands télescopes mis en réseau à ce jour sont de la classe 8-10 mètres.

La mesure de la visibilité complexe est affectée par la nature spectrale du signal détecté, par les défauts différentiels entre les trajets optiques et par les biais instrumentaux.

De très nombreux travaux sont mentionnés dans cet article et ne peuvent pas être tous cités en référence, faute de place. Nos lecteurs pourront retrouver leur description sur le site http://olbin.jpl.nasa.gov qui recense tous les articles publiés en astronomie. Ils peuvent aussi contacter les auteurs de l'article pour les aider à retrouver une référence précise. 
Tableau 1. Les principaux réseaux de télescopes.

\begin{tabular}{|c|c|c|c|c|}
\hline & Télescopes & Bases & Emplacement & Organisme \\
\hline VLTI [2] & $\begin{array}{l}4 \times 8 \mathrm{~m} \text { fixes } \\
4 \times 1,8 \mathrm{~m} \text { mobiles }\end{array}$ & $\begin{array}{l}130 m \\
200 m\end{array}$ & $\begin{array}{l}\text { Cerro Paranal } \\
\text { Chili }\end{array}$ & ESO \\
\hline CHARA [3] & $6 \times 1 \mathrm{~m}$ fixes & $330 \mathrm{~m}$ & Mt Wilson, Ca & U. of Georgia \\
\hline LBT & $\begin{array}{l}2 \times 8 \mathrm{~m} \\
\text { monture unique }\end{array}$ & $30 \mathrm{~m}$ & Mt Graham, Az & US, It, Ge \\
\hline Keck I & $2 \times 10 \mathrm{~m}$ fixes & $100 \mathrm{~m}$ & Mauna Kea, $\mathrm{Hi}$ & Keck/Nasa \\
\hline
\end{tabular}

Les défauts de planéité des fronts d'onde incidents affectent directement la qualité du signal interférométrique. Lorsque la recombinaison est effectuée en optique de volume, les fronts d'onde n'étant plus parfaitement co-planaires, il en résulte une chute de contraste des franges. Lorsque la recombinaison est réalisée en optique guidée, fibres optiques ou guides d'onde, ces défauts affectentl'efficacité d'injection dans les guides monomodes, dont l'étendue de faisceau correspond à la figure de diffraction de chaque télescope. On montre que l'effet résultant sur le rapport signal à bruit de la mesure interférométrique est équivalent dans les deux cas, réduisant d'autant la sensibilité.

Pour des observations effectuées depuis le sol, la turbulence atmosphérique est responsable des principaux défauts de planéité, réduisant la limite de résolution de chaque télescope à l'équivalent de la diffraction d'un diaphragme de diamètre $\rho$ caractérisant la turbulence. Ce diamètre $\rho$, linéairement dépendant de la longueur d'onde en première approximation, est de l'ordre du décimètre dans le visible, et voisin du mètre dans l'infrarouge.

L'effet de la turbulence se fait donc d'autant plus sentir que le diamètre des télescopes est grand ou que la longueur d'onde d'observation est courte. Les réseaux de petits télescopes $(D<1 \mathrm{~m})$ sont par conséquent peu sensibles à ces effets de turbulence dans le proche infrarouge, jusqu'à 2,4 $\mu \mathrm{m}$. Idéalement il est nécessaire de maintenir un rapport $D / \rho<3$ pour ne pas affecter de manière significative le contraste des franges.

\section{Les systèmes opérationnels}

Une quinzaine de réseaux interférométriques ont été construits depuis le milieu des années 70, suite à la proposition d'Antoine Labeyrie d'utiliser des réseaux de télescopes optiques sur des montures indépendantes, éventuellement mobiles. L'idée initiale proposée et démontrée sur le ciel à la fin du XIXe siècle, indépendamment en France par Fizeau et Stephan et auxÉtats-Unis par Michelson, faisait usage d'une monture unique, ce qui constituait une limite forte à la fois au regard des dimensions possibles et en termes de rigidité et de stabilité de ces structures.

À ce jour on retiendra pour notre propos quatre systèmes principaux en cours d'utilisation dont les principales caractéristiques sont données dans le tableau 1. Les équipes françaises sont fortement impliquées pour la réalisation des instruments de recombinaison et leur exploitation scientifique en particulier avec le VLTI de I'ESO [2] (figure 1) et le réseau CHARA de la Georgia State University [3]

La section suivante détaillera l'instrumentation installée sur ces réseaux.

\section{Quelle instrumentation, pour quelle science?}

Après deux décennies de développement instrumental, depuis le début des années 2000 plusieurs réseaux fournissent aux astronomes des données astrophysiques de manière routinière. Un nombre croissant d'articles sont publiés sur les résultats de l'interférométrie, de plus en plus consacrés aux résultats astrophysiques (figure 2).

Des instruments spécifiques sont maintenant construits en fonction du type d'observations astrophysiques considérées.

\section{Imagerie}

L'objectif astrophysique est dans ce cas l'étude de la morphologie des objets observés. Par exemple dans le cas d'étoiles

\section{FILTRES \\ Accordables \\ Option Ultrafin sur XTA-50 et XTM-50}

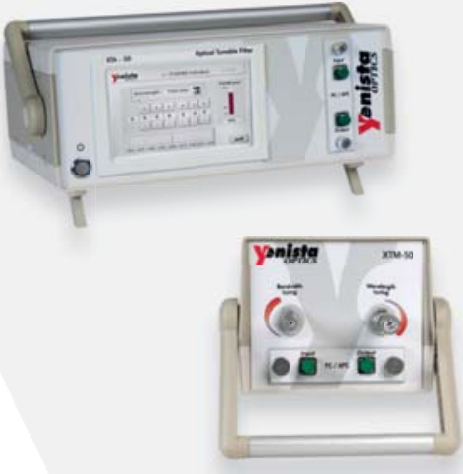

FWHM mini : 32 pm Pente : $800 \mathrm{~dB} / \mathrm{nm}$

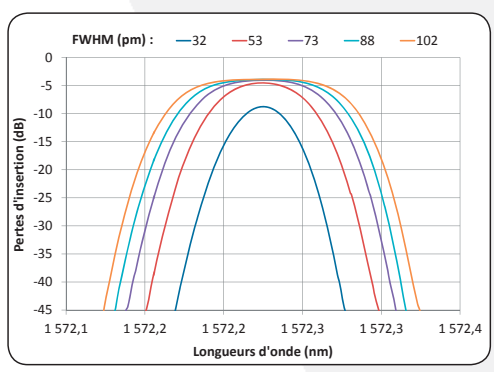

\section{Gamme complète}

Filtres XT :

Bande SCL ou O, SMF ou

PMF, automatique ou manuel

\section{Filtres WS :}

Passe-bande ou coup-bande

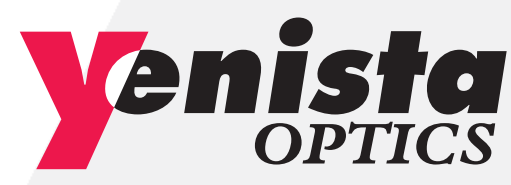
Tél. : +33 (0)2 96483716 sales-emea@yenista.com www.yenista.com




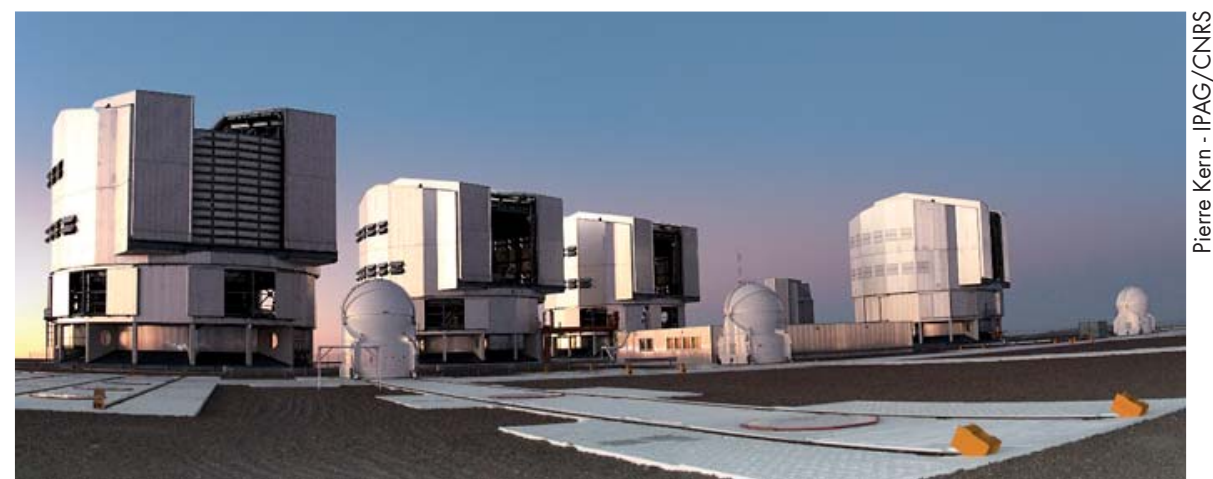

Figure 1. Le VLTI (Very Large Telescope Interferometer) [2] installé sur le Cerro Paranal au Chili dans le désert d'Atacama.

jeunes en formation on cherchera à analyser la structure d'un disque de matière autour de l'objet central, d'un jet, éventuellement de détecter la présence d'une ou plusieurs planètes en orbite. Dans le cas d'étoiles géantes on cherche à obtenir l'image de leur surface. Des analyses de structures de galaxies proches sont aussi considérées. Les instruments spécifiquement dédiés à ce type de mesures sont MI6/MIRC en opération sur CHARA depuis 2009, tirant profit dans le proche infrarouge des six télescopes du réseau. De même PIONIER installé sur le VLTI fin 2010 utilise quatre télescopes pour ce type d'applications dans le proche infrarouge (1,5 à 2,4 $\mu \mathrm{m})$. L'instrument MATISSE, en cours de construction par un consortium européen piloté par l'Observatoire de la Côte d'Azur fera quant à lui le même type d'observation dans l'infrarouge moyen (3 à $20 \mu \mathrm{m}$ ) sur le VLTI.

Pour ces instruments les caractéristiques critiques sont la sensibilité, contrainte par la transmission de l'instrument et le diamètre des télescopes, la résolution angulaire, contrainte par la longueur des lignes de base, et la fidélité de reconstruction des images, contrainte par le nombre de lignes de base accessibles, c'est-à-dire par le nombre de télescopes recombinés.

\section{Astrométrie : mesure de position à très haute précision}

Parmi les enjeux scientifiques on retiendra ici les applications où il est essentiel de mesurer le déplacement absolu d'une étoile avec une précision de quelques microsecondes d'arc. C'est le cas du déplacement induit par la présence d'une ou plusieurs planètes extrasolaires autour de l'étoile hôte. C'est l'un des objectifs visés par PRIMA en cours de caractérisation au VLTI. Cet instrument permet une observation simultanée de deux étoiles au moyen de deux télescopes dans le de la base de données OLBIN, voir [1]). proche infrarouge (1,5 à 2,4 $\mu \mathrm{m})$. C'est le cas aussi de la mesure de l'influence d'un objet très massif, trou noir en particulier, sur la trajectoire des étoiles dans sa proximité. GRAVITY en cours de construction dans un consortium piloté par le MPE (Max-Planck-Institut für extraterrestrische Physik) à Garching en Allemagne est spécifiquement conçu pour étudier l'influence du trou noir au centre de notre Galaxie.

Ces instruments réalisent une mesure de phase différentielle entre un objet fournissant la référence de phase et l'objet astrophysique étudié. De ce fait la différence entre les trajets optiques correspondants à ces deux objets doit être mesurée et calibré minutieusement. Pour atteindre les performances requises, il est indispensable d'utiliser une métrologie interne à l'instrument utilisant autant que faire se peut les mêmes chemins optiques que les

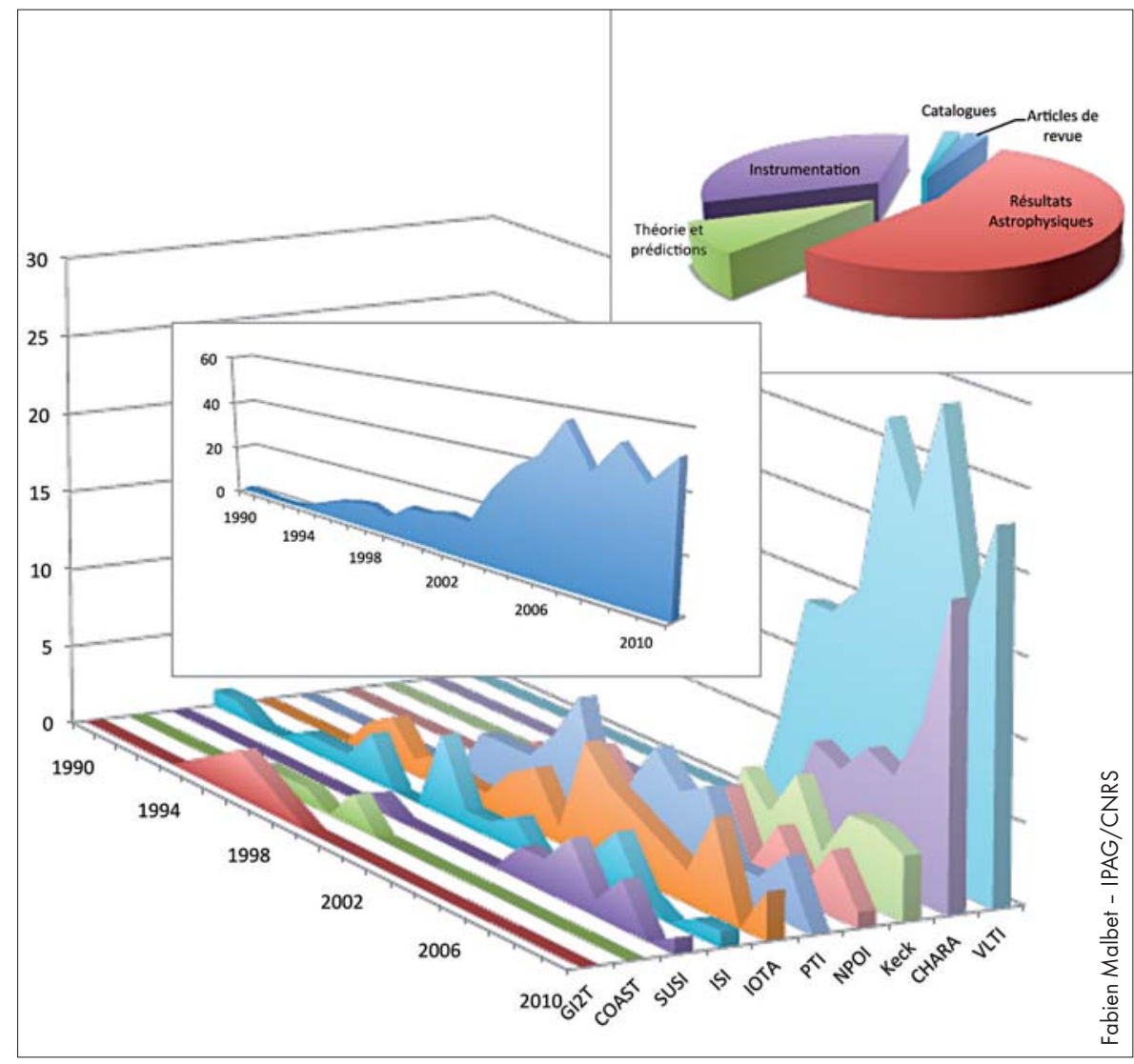

Figure 2. Statistiques des articles publiés en interférométrie sur les résultats astrophysiques obtenus. Un premier histogramme donne l'évolution des publications pour l'ensemble des interféromètres, le second histogramme présente cette statistique par interféromètre. Le camembert donne la répartition des articles publiés par la discipline en fonction de l'objectif de la publication : instrumentation, résultats astrophysiques, articles de revue, catalogues ou prédictions (figure transmise par Fabien Malbet/IPAG à partir 
faisceaux provenant des objets stellaires. Ici l'enjeu le plus critique est une mesure de la différence de phase très précise lenviron $5 \mathrm{~nm}$ pour atteindre 5 micro-arcsec avec une base de 100 mètres dans le proche infrarouge).

\section{Spectro-interférométrie}

L'objectif est d'obtenir une mesure de la visibilité complexe en fonction de la longueur d'onde et/ou de l'état de polarisation. Les instruments sont des spectromètres à haute résolution permettant $d^{\prime}$ 'analyserfinement les raies d'émission et d'absorption des objets observés. L'étude des raies atomiques et moléculaires donne accès aux conditions physiques à la surface de l'objet (température, pression, composition chimique). Grâce à l'effet Doppler, la position et la forme des raies renseignent sur la cinématique, par exemple la présence d'un disque en rotation, d'une enveloppe en expansion, ou de jets de matière collimatés. En pratique, des résolutions spectrales comprises entre 1000 et 100000 sont nécessaires. L'ensemble du spectre optique (de 0,4 à $20 \mu \mathrm{m}$ ) présente un intérêt. L'accès au visible est particulièrement important.

AMBER a été spécifiquement construit pour ce type d'observations sous la direction d'astronomes de Nice et de Grenoble. Depuis 2004, cet instrument permet la recombinaison dans le proche infrarouge (1,5 à 2,4 $\mu \mathrm{m})$ des faisceaux provenant de trois télescopes du VLTI. Un instrument similaire, ASTRA, est actuellement installé sur l'interféromètre à deux télescopes du KECK. L'instrument VEGA, construit par une équipe de Nice, recombine 3 ou 4 télescopes du réseau CHARA et délivre une résolution spectrale de 30000 dans le visible $(0,5 \mu \mathrm{m})$.

Lorsque le rapport signal sur bruit est dominé par le détecteur, en particulier dans le proche infrarouge, il devient indispensable de pouvoir augmenter les temps de pose (seconde, minutes) imposant de savoir stabiliser les franges d'interférence. Cette stabilisation se fait au moyen d'un suiveur de frange, asservissement agissant à la manière d'une optique adaptative, qui mesure en temps réel (environ $1 \mathrm{kHz}$ l la position de la frange centrale. Cet asservissement agit sur la ligne à retard pour corriger les erreurs de position dues à la turbulence de l'atmosphère ou aux vibrations instrumentales. Dans le cas de détecteurs avec une capacité de comptage de photons, cet aspect devient un peu moins critique, en particulier dans le domaine visible.

\section{Interférométrie d'annulation}

Les programmes d'interférométrie les plus ambitieux visent la détection de signatures de vie sur des planètes en dehors de notre système solaire. Outre une détection à très haute résolution angulaire, pour analyser un objet extrêmement proche de son étoile hôte, il est nécessaire de réaliser une mesure à très fort contraste pour extraire la signature des composants chimiques recherchés sur ces planètes par rapport au flux stellaire. Les meilleures

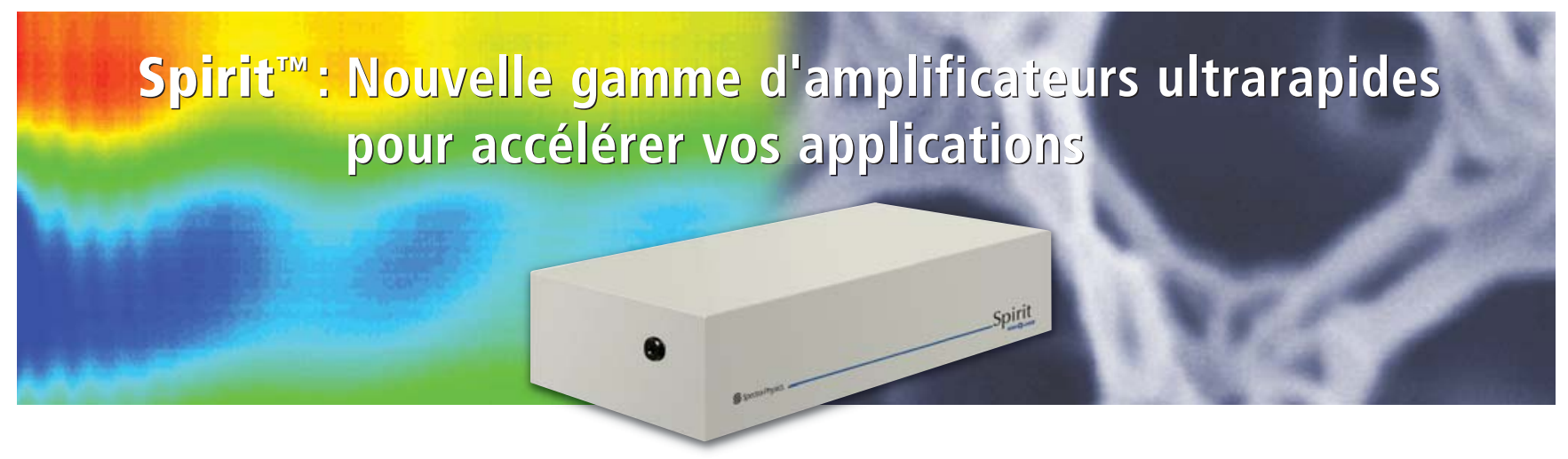

Spectra-Physics présente sa nouvelle gamme d'amplificateurs ultra-rapides tout-en-un Spirit' ${ }^{\mathrm{T} M}$ à haut taux de répétitivité et réglable. Basée sur la technologie de pompage direct par diodes, développée par High Q Lasers (désormais intégrée à Spectra-Physics), l'architecture simple et novatrice de la gamme Spirit délivre des impulsions femtosecondes nécessaires au micro-usinage de composants médicaux et autres matériaux, ainsi qu'à la nanostructuration et aux applications scientifiques à résolution temporelle sélectionnée. Avec les amplificateurs Spirit, accélérez vos applications et obtenez des résultats rapidement.

Acteur majeur de l'innovation, Spectra-Physics se positionne une fois de plus comme le leader du marché des solutions ultra-rapides en proposant la gamme de technologies la plus vaste, les champs d'application les plus variés et la base installée la plus étendue du secteur.

Pour plus d'informations sur la gamme Spirit, consultez www.newport.com/spirit ou appelez-nous.

MICRO-CONTROLE Spectra-Physics S.A.S

1 , rue Jules Guesde - Bâtiment B

Zl. du Bois de l'Épine - BP189

91006 Évry CEDEX
Spectra-Physics. \#1 in Ultrafast.

Expertise - Innovation - Results

Tél. : 01.60.91.68.68
Fax: 01.60.91.68.69

e-mail: france@newport.com
S Spectra-Physics.

une marque de Newport Corporation

(c) 2012 Newport Corporation. 
conditions sont atteintes dans l'infrarouge moyen aux alentours de $10 \mu \mathrm{m}$ où le contraste nécessaire est de 10'. La technique d'interférométrie d'annulation a été proposée avec cet objectif. Dans ce cas un déphasage de $\pi$ est généré entre les faisceaux provenant de l'étoile centrale et de la planète. Ainsi l'étoile se trouve centrée sur une frange noire du système interférométrique qui agit à la manière d'un coronographe, alors que la planète est placée sur une frange blanche. Des premiers démonstrateurs ont été réalisés en laboratoire et sur le ciel, pour franchir les étapes intermédiaires, mais les performances ultimes ne seront accessibles que depuis l'espace.

L'efficacité de ce système d'annulation est directement liée à la profondeur de la frange noire que l'on est capable d'atteindre et elle sera très fortement contrainte par la qualité instrumentale sur l'ensemble des trajets optiques. II sera indispensable de contrôler avec le plus grand soin les défauts de polarisation, de chromatisme, de différence d'éclairement entre les télescopes et de contrôler la différence de marche entre les faisceaux. Il a été identifié suite aux travaux réalisés conjointementà l'IAS à Orsay età l'Observatoire de Paris, qu'un filtrage modal est précieux pour atteindre le niveau de planéité requis. En optique de volume la spécification sur la planéité est de $\lambda / 6000$ pour atteindre un contraste de $10^{6}$. L'utilisation d'un filtrage modal par un guide d'onde ou une fibre monomode réduit cette contrainte à $\lambda / 60$.

\section{Quelques technologies clés}

La section précédente a mis en évidence comment la structure même de l'interféromètre conditionne les performances attendues, en particulier par la taille et le nombre des télescopes, ainsi que par leur espacement. Par ailleurs pour un réseau de télescopes donné, les performances vont aussi être contraintes par l'instrument de recombinaison luimême. Parmi les limitations importantes on peut noter la sensibilité (turbulence atmosphérique, sensibilité des détecteurs, transmission du système) et la com- plexité du système imposée par exemple par un nombre croissant de télescopes ou par les fonctions requises par les modes d'observations, la dispersion chromatique dans les liens optiques entre les télescopes, incluant les lignes à retard. Nous abordons ici les travaux menés actuellement en France pour réduire les limitations les plus critiques.

\section{La détection}

$C^{\prime}$ est sur ce point que les premières améliorations sont attendues. Pour le proche infrarouge, les détecteurs actuellement disponibles ne permettent pas encore d'atteindre en régime de faible flux la limite du bruit de photons. Ils sont encore limités par leur bruit de lecture. Dans le cas des applications interférométriques, ils sont aussi limités par la vitesse de lecture : ceci empêche d'échantillonner le temps de cohérence des franges d'interférence, proche d'une dizaine de millisecondes, imposé par la turbulence atmosphérique. L'arrivée de nouvelles matrices de photodiodes à avalanche en $\mathrm{HgCdTe} \mathrm{sera} \mathrm{un} \mathrm{pas} \mathrm{décisif} \mathrm{dans} \mathrm{ce} \mathrm{sens.}$ Des développements sont en cours dans le projet RAPID dirigé par l'IPAG incluant Sofradir et le LIR pour les aspects technologiques, I'IPAG, le LAM et l'Onera pour les aspects applicatifs. L'objectif est d'atteindre un bruit de lecture inférieur à $3 \mathrm{e}-/ \mathrm{px}$ par lecture et une cadence trame supérieure à $1 \mathrm{kHz}$.

Dans le domaine du visible, les détecteurs disponibles fonctionnant en mode de comptage de photons souffrent d'un rendement quantique limité à celui des photocathodes de leurs systèmes intensificateurs, avec des valeurs rarement supérieures à $30 \%$. Par ailleurs la vitesse de comptage de ces détecteurs en limite l'utilisation en condition de fort flux, imposant d'atténuer le flux incident au prix d'une perte du rapport signal sur bruit. Des développements sont menés à l'Observatoire de la Cote d'Azur dans ce domaine.

\section{Optique adaptative}

Sur les interféromètres utilisant les plus grands télescopes, la sensibilité est significativement augmentée par l'utilisation de systèmes d'optique adaptative au foyer de chaque télescope. Ils permettent soit d'optimiser l'injection dans des systèmes d'optique guidée, soit d'obtenir une meilleure planéité des fronts d'onde à superposer en optique de volume. De tels systèmes sont opérationnels sur les télescopes de 8 mètres du VLTI ou de 10 mètres du Keck-l. L'installation de dispositifs d'optique adaptative pour les télescopes de 1,8 mètre du VLTI et les télescopes de 1 mètre de CHARA est actuellement à l'étude. Les systèmes d'optique adaptative maîtrisés à ce jour répondent à ce besoin. L'enjeu est davantage dans la mise en œuvre du système complet, incluant l'ajustement des paramètres en fonction des applications et la synchronisation des modules d'asservissement.

\section{Le transport des faisceaux}

Pour les réseaux interférométriques en cours d'exploitation, le transport des faisceaux et les lignes à retard qui compensent les différences de chemins optiques générés par le déplacement des étoiles, sont réalisés par un train de miroirs. La longueur de propagation, en général supérieure à 100 mètres, impose le diamètre des miroirs pour ne pas être limité par la diffraction. Lorsque ce train optique fonctionne à l'air libre, la dispersion chromatique de l'air dégrade la mesure. Une solution consiste à opérer en atmosphère contrôlée idéalement sous vide, ce qui devient difficile à mettre en œuvre et très onéreux pour des réseaux étendus à grand nombre de télescopes. Une autre solution proposée par une équipe $d u$ LESIA à Meudon et de Xlim à Limoges consiste à utiliser des fibres optiques (programme OHANA). La difficulté réside ici dans la disponibilité de fibres optiques à dispersion quasi nulle pour les gammes de longueur d'onde considérées, en particulier au-delà de $2 \mu \mathrm{m}$. Jusqu'à 2,5 $\mu \mathrm{m}$ des solutions à base de verre fluoré sont considérées. Des solutions à base de fibres à cristaux photoniques ont été analysées par l'équipe XLIM.

\section{L'optique intégrée, un outil précieux pour une instrumentation complexe}

La complexité instrumentale croît très rapidement avec le nombre de télescopes, apportant des contraintes croissantes 


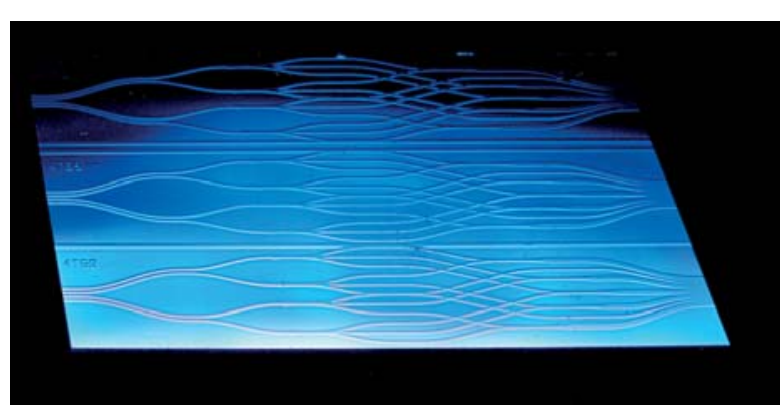

sur la mise en œuvre. Les alignements optiques deviennent plus lourds et difficiles à maintenir aux meilleures performances. Les montages interférométriques sont aussi très sensibles aux contraintes environnementales, température et vibrations en particulier.

L'optique intégrée offre une solution élégante pour réduire l'effet de ces contraintes. L'IPAG à Grenoble en lien avec I'IMEP initialement puis avec le LETI a développé des puces optiques de quelques centimètres carrés au plus, contenant l'ensemble du module de recombinaison pour deux à quatre télescopes dans le proche infrarouge jusqu' à 2,5 $\mu \mathrm{m}$. Les contraintes d'alignement se réduisent à l'injection dans les guides d'entrée via des fibres optiques monomodes. Un tel module très compact assure une excellente stabilité favorable à une mesure de phase robuste. Ce concept a été démontré dès le début des années 2000 sur l'interféromètre IOTA au Mt Hopkins, en Arizona d'abord avec deux télescopes. Cette démonstration a convaincu dès 2002 l'équipe américaine d'utiliser ce principe pour leur instrument de recombinaison à trois télescopes dédié à la bande $H(1,5$ à 1,8 $\mu \mathrm{m})$. Celui-ci a permis une exploitation intensive pendant une demidouzaine d'années sur des objectifs astrophysiques.

Sur la lancée de cette expérience, la technologie a été retenue pour GRAVITY au VLTI, à la fois pour l'instrument scientifique de recombinaison et pour son système de suivi de frange. Des développements technologiques conséquents entre I'IPAG et le LETI ont été menés pour mettre au point des puces avec la meilleure transmission dans le domaine d'observation de GRAVITY, entre 2 et 2,4 4 m (bande K). Ces développements s'appuient sur des

2
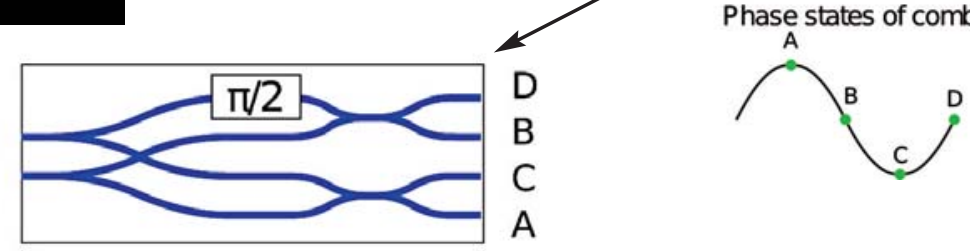

A

Figure 3. Composant de recombinaison pour quatre télescopes utilisé sur PIONIER pour la bande de 1,5 à 1,8 $\mu \mathrm{m}$, en mode $A B C D$ fournissant quatre points de mesure par frange. Les quatre entrées alimentées par des fibres optiques maintenues dans un V-groove sont divisées en trois parties égales pour contribuer au codage de l'ensemble des lignes de base accessibles ( 6 lignes de bases dans ce cas). Une seconde division en 2 voies est effectuée pour introduire sur l'une d'elle, un déphasage de $\pi / 2$. Les 4 sorties ainsi obtenues par ligne de base permettent de mesurer 4 états de phase en quadrature.

technologies de silice gravée sur silicium. En avance de phase, anticipant la mise en opération de GRAVITY, I'IPAG a proposé à l'ESO un instrument visiteur permettant la recombinaison de quatre télescopes dans la bande $\mathrm{H}$ et prochainement dans la bande K. Cet instrument d'équipe, installé au VLTI à l'automne 2009, fournit depuis des données astrophysiques de premier plan. La souplesse d'utilisation de cette technique permet aussi de changer de schéma de recombinaison simplement en changeant la puce optique. Dans le cas de PIONIER ceci permet de passer d'un mode de recombinaison avec un codage $A B C D$ lquatre points de mesure par frange) à un codage $A C$ (deux points de mesure par frange) ou prochainement de la bande H à la bande K (figure 3).

Pour étendre le domaine de longueur d'onde d'application de cette technique, I'IPAG mène des développements complémentaires. L'un des développements en cours utilise des substrats en niobate de lithium visant des applications jusque vers $5 \mu \mathrm{m}$ en lien avec Femto-ST à Besançon. D'autres développements sont menés en utilisant des verres de chalcogénure pour de plus grandes longueurs d'onde, jusqu' à $15 \mu \mathrm{m}$, avec des partenaires étrangers (université Heriot Watt à Edimbourg) et français (LPHC/Saint-Etienne, LVC)
Rennes et Femto-ST/Besançon). De premiers résultats très encourageants ont été obtenus récemment en laboratoire avec un re-combinateur à trois télescopes testé dans la gamme 8 à $11 \mu \mathrm{m}$.

\section{En conclusion}

L'interférométrie a maintenant atteint un niveau de maturité qui permet son utilisation pour produire des résultats astrophysiques à très haute résolution angulaire. L'objectif est maintenant de travailler à l'amélioration des performances, par la mise en œuvre de nouvelles technologies d'une part, et par une minutieuse optimisation système d'autre part.

\section{Références}

[1] Une bibliographie complète sur l'ensemble des travaux mentionnés est disponible sur le site OLBIN de I'Union Astronomique Internationale : http://olbin.jpl.nasa.gov/

[2] Le VLTI de I'ESO : http://www.eso.org/sci/ facilities/paranal/telescopes/vlti/index.html

[3] The CHARA Array of Georgia State University: http://www.chara.gsu.edu/CHARA/ 